\title{
Outcomes of Anatomical versus Functional Testing for Coronary Artery Disease
}

\section{Citation}

Douglas, Pamela S., Udo Hoffmann, Manesh R. Patel, Daniel B. Mark, Hussein R. Al-Khalidi, Brendan Cavanaugh, Jason Cole, et al. 2015. "Outcomes of Anatomical Versus Functional Testing for Coronary Artery Disease." N Engl J Med 372 (14) (April 2): 1291-1300. doi:10.1056/ nejmoa1415516.

\section{Published Version}

doi:10.1056/NEJMoa1415516

\section{Permanent link}

http://nrs.harvard.edu/urn-3:HUL.InstRepos:29048899

\section{Terms of Use}

This article was downloaded from Harvard University's DASH repository, and is made available under the terms and conditions applicable to Open Access Policy Articles, as set forth at http:// nrs.harvard.edu/urn-3:HUL.InstRepos:dash.current.terms-of-use\#OAP

\section{Share Your Story}

The Harvard community has made this article openly available.

Please share how this access benefits you. Submit a story.

\section{Accessibility}




\title{
Abstract
}

BACKGROUND-Many patients have symptoms suggestive of coronary artery disease (CAD) and are often evaluated with the use of diagnostic testing, although there are limited data from randomized trials to guide care.

\begin{abstract}
METHODS-We randomly assigned 10,003 symptomatic patients to a strategy of initial anatomical testing with the use of coronary computed tomographic angiography (CTA) or to functional testing (exercise electrocardiography, nuclear stress testing, or stress echocardiography). The composite primary end point was death, myocardial infarction, hospitalization for unstable angina, or major procedural complication. Secondary end points included invasive cardiac catheterization that did not show obstructive CAD and radiation exposure.
\end{abstract}

RESULTS-The mean age of the patients was $60.8 \pm 8.3$ years, $52.7 \%$ were women, and $87.7 \%$ had chest pain or dyspnea on exertion. The mean pretest likelihood of obstructive CAD was $53.3 \pm 21.4 \%$. Over a median follow-up period of 25 months, a primary end-point event occurred in 164 of 4996 patients in the CTA group (3.3\%) and in 151 of 5007 (3.0\%) in the functional-testing

\footnotetext{
Copyright (C) 2015 Massachusetts Medical Society.

Address reprint requests to Dr. Douglas at Duke University School of Medicine, 7022 North Pavilion, Duke University Medical Center, P.O. Box 17969, Durham, NC 27715, or at pamela.douglas@duke.edu.

A complete list of investigators in the Prospective Multicenter Imaging Study for Evaluation of Chest Pain (PROMISE) is provided in the Supplementary Appendix, available at NEJM.org.

No other potential conflict of interest relevant to this article was reported.

Disclosure forms provided by the authors are available with the full text of this article at NEJM.org.
} 
group (adjusted hazard ratio, $1.04 ; 95 \%$ confidence interval, 0.83 to $1.29 ; \mathrm{P}=0.75$ ). CTA was associated with fewer catheterizations showing no obstructive CAD than was functional testing ( $3.4 \%$ vs. $4.3 \%, \mathrm{P}=0.02$ ), although more patients in the CTA group underwent catheterization within 90 days after randomization ( $12.2 \%$ vs. $8.1 \%)$. The median cumulative radiation exposure per patient was lower in the CTA group than in the functional-testing group (10.0 mSv vs. 11.3 $\mathrm{mSv}$ ), but $32.6 \%$ of the patients in the functional-testing group had no exposure, so the overall exposure was higher in the CTA group (mean, $12.0 \mathrm{mSv}$ vs. $10.1 \mathrm{mSv}$; $\mathrm{P}<0.001$ ).

CONCLUSIONS-In symptomatic patients with suspected CAD who required noninvasive testing, a strategy of initial CTA, as compared with functional testing, did not improve clinical outcomes over a median follow-up of 2 years. (Funded by the National Heart, Lung, and Blood Institute; PROMISE ClinicalTrials.gov number, NCT01174550.)

New-onset, stable chest pain is a common clinical problem that results in approximately 4 million stress tests annually in the United States in ambulatory patients without diagnosed heart disease. ${ }^{1}$ Despite advances in cardiac testing, there is scant information on healthrelated outcomes and little consensus about which noninvasive test is preferable. ${ }^{2-4}$ As a result, current patterns of care have been questioned, including the testing of very-low-risk populations ${ }^{5}$ and the catheterization of patients who do not have obstructive coronary artery disease (CAD). ${ }^{6-8}$

The development of coronary computed tomographic angiography (CTA) and its application in this context has the potential to reduce unnecessary invasive testing and improve outcomes, owing to its substantially higher accuracy, as compared with functional testing, and its unique ability to detect prognostically important but nonobstructive CAD. ${ }^{9-13}$

However, the relative impact of data from noninvasive anatomical testing versus functional testing on subsequent management and clinical outcomes is not known. ${ }^{14,15}$

The objective of the Prospective Multicenter Imaging Study for Evaluation of Chest Pain (PROMISE) was to compare health outcomes in patients who presented with new symptoms suggestive of CAD that required further evaluation and who were randomly assigned to an initial strategy of anatomical testing with the use of CTA or to functional testing. The primary hypothesis of the study was that the clinical outcomes in patients assigned to anatomical testing with the use of CTA would be superior to those in patients assigned to functional testing.

\section{METHODS STUDY DESIGN}

This trial used a pragmatic comparative effectiveness design that has been described previously. ${ }^{16}$ The study was conducted with fidelity to the protocol (available with the full text of this article at NEJM.org). The study protocol was approved by the local or central institutional review board at each coordinating center and at each of the 193 enrolling sites in North America. The study sites included those with expertise in the fields of cardiology, primary care, radiology, and anesthesia and represented both the community and academia. 
The study was supported solely by grants from the National Heart, Lung, and Blood Institute (NHLBI), and there were no agreements regarding data confidentiality. The authors coordinated the trial, managed the database, independently performed the analyses, and wrote the drafts of the manuscript. Together with NHLBI representatives, they designed the trial, oversaw study conduct and reporting, and take responsibility for the accuracy and completeness of data analyses.

\section{STUDY PARTICIPANTS}

The study participants were symptomatic outpatients without diagnosed CAD whose physicians believed that nonurgent, noninvasive cardiovascular testing was necessary for the evaluation of suspected CAD. Additional inclusion criteria were an age of more than 54 years (in men) or more than 64 years (in women) or an age of 45 to 54 years (in men) or 50 to 64 years (in women) with at least one cardiac risk factor (diabetes, peripheral arterial disease, cerebrovascular disease, current or past tobacco use, hypertension, or dyslipidemia). Exclusion criteria were an unstable hemodynamic status or arrhythmias that required urgent evaluation for suspected acute coronary syndrome, a history of CAD or evaluation for CAD within the previous 12 months, or clinically significant congenital, valvular, or cardiomyopathic heart disease, or any reason that the patient could not be randomly assigned to either group safely (Table S1 in the Supplementary Appendix, available at NEJM.org).

\section{STUDY PROCEDURES}

After providing written informed consent, participants were randomly assigned to either the CTA group or the functional-testing group, with stratification according to study site and according to the choice, as indicated before randomization by the managing caregiver, site investigator, or other authorized personnel, of the intended functional test if the patient were to be assigned to that study group. ${ }^{16}$ Tests were performed and interpreted by local physicians who made all sub-sequent clinical decisions. Appropriate medical therapy was encouraged, and educational materials were provided to patients and providers (see the Supplementary Appendix). Follow-up visits were performed at 60 days at the study sites and centrally by means of telephone or mail at 6-month intervals after randomization, for a minimum of 1 year.

Enrollment began on July 27, 2010, and was completed on September 19, 2013. On April 30,2013 , the protocol was amended to require a minimum follow-up of 1 year, rather than 2 years. The decision was based on budgetary limitations, after careful consideration of the importance of the 12-month post-test period for judging the effect of the diagnostic testing strategies. All the patients were followed until October 31, 2014.

\section{DIAGNOSTIC TESTING}

Before enrollment, the PROMISE Diagnostic Testing Coordinating Center certified the study sites with respect to all the testing methods, including compliance with professional society guidelines regarding the quality of test equipment, staff and physician experience, and image-acquisition protocols. Functional testing included exercise electrocardiography (ECG), exercise or pharmacologic nuclear stress testing, and stress echocardiography. 
Anatomical testing was contrast-enhanced CTA performed with the use of a 64-slice or greater multidetector CT scanner.

\section{EFFECTIVENESS AND SAFETY END POINTS}

The primary end point was a composite of major cardiovascular events that included death from any cause, myocardial infarction, hospitalization for unstable angina, and major complication of cardiovascular procedures or diagnostic testing (stroke, major bleeding, renal failure, or anaphylaxis) that occurred within 72 hours, over the entire follow-up period for each patient ${ }^{16}$ (end-point definitions are provided in Table S2 in the Supplementary Appendix). Secondary end points included a composite of the primary end point or invasive catheterization showing no obstructive CAD, other combinations of the components of the primary end point, invasive cardiac catheterization showing no obstructive CAD, and cumulative radiation exposure; the latter two end points were determined at 90 days. Members of an independent clinical-events committee adjudicated all primary and secondary end-point events in a blinded fashion on the basis of standard, prospectively determined definitions. ${ }^{17}$

Cumulative radiation exposure was defined as radiation exposure related to all the cardiovascular testing or procedures performed within 90 days after randomization, including CTA, nuclear stress testing, and invasive coronary angiography or angioplasty. Radiation exposure was measured in millisieverts and was calculated or imputed with the use of standard methods. ${ }^{18-20}$ No obstructive CAD on invasive catheterization was defined as an estimated absence of stenosis of $50 \%$ or more, as interpreted by the study-site staff, in any major epicardial vessels, including side branches of at least $2 \mathrm{~mm}$ in diameter, on the first cardiac catheterization performed within 90 days after randomization. If the study-site report regarding invasive cardiac catheterization was inconclusive, catheterization films were reviewed for a visual assessment of CAD.

\section{STATISTICAL ANALYSIS}

We estimated that enrollment of 10,000 patients would provide the study with $90 \%$ power to detect a relative reduction of $20 \%$ in the primary end point in the CTA group, as compared with the functional-testing group, assuming an event rate of $8 \%$ in the functional-testing group at 2.5 years and a $3 \%$ loss to follow-up, at an alpha significance level of 0.05 . In addition, the study was planned so that in the event of a nonsignificant result in the comparison for superiority, there was sufficient power for a prespecified noninferiority assessment to rule out a $10 \%$ relative increase in the risk of the primary end point in the CTA group, assuming that anatomical testing was better than functional testing by $10 \%$. Statistical comparisons of the diagnostic testing strategies were performed according to the randomization assignment. Since all the patients in the study were to be followed for a minimum of 1 year, secondary analyses of the primary and secondary end points were performed on the basis of 1-year outcomes.

Statistical comparisons of the two randomized groups were based on a time-to-first-event analysis that used the Cox proportional-hazards model. ${ }^{21}$ To account appropriately for heterogeneity among the study patients, comparisons were adjusted for a prespecified set of 
baseline covariates, including age, sex, CAD risk equivalent (history of diabetes, peripheral arterial disease, or cerebrovascular disease), and the prespecification of the intended functional test if the patient were to be randomly assigned to the functional-testing group. Relative risks were expressed as adjusted hazard ratios with associated $95 \%$ confidence intervals and were derived from the Cox model. Cumulative event rates were calculated for each randomized group as a function of the time from randomization with the use of the Kaplan-Meier method. ${ }^{22}$ The Cox model was also used to assess the consistency of the effects of the diagnostic testing strategy by testing for interactions between the diagnostic strategy and baseline characteristics that were prespecified for subgroup analysis. Radiation exposure was compared between the randomized groups with the use of the Wilcoxon ranksum test.

No interim analyses of the primary and secondary end points were performed; therefore, the alpha significance level in the final primary analysis was 0.05 . We performed all the comparisons using two-sided significance tests. All the analyses were performed with the use of SAS software, version 9.2 or higher (SAS Institute).

\section{RESULTS STUDY POPULATION}

The study population consisted of 10,003 patients (Fig. 1). The median follow-up time was 25 months (interquartile range, 18 to 34), with a maximum follow-up time of 50 months. Complete follow-up of at least 12 months was obtained for 9350 participants (93.5\%).

\section{CHARACTERISTICS AT BASELINE}

The mean age of the patients was $60.8 \pm 8.3$ years, 5270 of the 10,003 patients $(52.7 \%)$ were women, and 2248 of the 9941 patients with data (22.6\%) belonged to a racial or ethnic minority group (Table 1). The study population had a substantial burden of cardiovascular risk factors: $21.4 \%$ of the patients had diabetes, $65.0 \%$ had hypertension, $51.1 \%$ were past or current tobacco users, $67.7 \%$ had dyslipidemia, and $32.1 \%$ had a family history of premature CAD. Patients had a mean of 2.4 of these five risk factors; only $2.6 \%$ of the patients were enrolled in the study by meeting the age criterion alone. A CAD risk equivalent (diabetes, peripheral vascular disease, or cerebrovascular disease) was present in 2531 patients (25.3\%). The assessment of cardiac risk, which was calculated according to the 2013 atherosclerotic cardiovascular disease risk score from the American College of CardiologyAmerican Heart Association guidelines, showed that 6697 of 9901 patients (67.6\%) had a 10 -year risk of events of $7.5 \%$ or higher. ${ }^{24}$ The use of cardiovascular medications was common (Table 1).

All the patients (except 7 patients for whom records were missing) were symptomatic, with 8762 of the 9996 patients (87.7\%) reporting either chest pain (7272 [72.7\%]) or dyspnea on exertion $(1490[14.9 \%])$ as the primary symptom. In the remaining $12.3 \%$ of patients, the primary symptom was (in descending order of frequency) fatigue or weakness, arm or shoulder pain, palpitations, dizziness or light-headedness, or neck or jaw pain. Chest pain was described as aching or crushing pain in $70.7 \%$ of the patients and was relieved by 
nitroglycerin or rest in $33.3 \%$ of the patients. The mean pretest likelihood of obstructive disease according to a combined Diamond and Forrester and Coronary Artery Surgery Study model was $53.3 \pm 21.4 \% .^{2}$

\section{INITIAL TESTING}

Among the 4996 participants randomly assigned to a CTA strategy, 4686 (93.8\%) had a CTA as the initial test, 145 (2.9\%) had a functional test, and 156 (3.1\%) had no test (Fig. 1). A total of 9 patients in the CTA group proceeded directly to catheterization. Among the 5007 patients randomly assigned to a functional-test strategy, $4692(93.7 \%)$ had a stress test, $47(0.9 \%)$ had a CTA, $246(4.9 \%)$ had no test, and 2 patients had the test before randomization. A total of 20 patients in the functional-testing group proceeded directly to catheterization.

Among the 4837 patients from either group who underwent a functional test, 3263 (67.5\%) underwent nuclear stress testing, 1083 (22.4\%) stress echocardiography, and 491 (10.2\%) exercise ECG; $29.4 \%$ of the stress tests were pharmacologic. The study sites reported their interpretation of the initial test as positive for CAD in 517 of 4840 patients (10.7\%) in the CTA group and in 556 of 4759 (11.7\%) in the functional-testing group (Table S3 in the Supplementary Appendix lists the criteria for positive tests).

\section{OUTCOME MEASURES}

During follow-up, 164 patients (3.3\%) in the CTA group and $151(3.0 \%)$ in the functionaltesting group had a primary end-point event (hazard ratio, $1.04 ; 95 \%$ confidence interval [CI], 0.83 to 1.29; $\mathrm{P}=0.75$ ) (Table 2 and Fig. 2). The upper limit of the $95 \%$ confidence interval (1.29) exceeded the conservative prespecified noninferiority margin of 1.10 . Results were also not significant for the secondary end point of the composite of the primary end point plus invasive cardiac catheterization showing no obstructive CAD, which occurred in 332 patients $(6.6 \%)$ in the CTA group and in $353(7.1 \%)$ in the functional-testing group (hazard ratio, $0.91 ; 95 \%$ CI, 0.78 to $1.06 ; \mathrm{P}=0.22$ ) (Fig. S1 in the Supplementary Appendix). Other combinations of the primary end-point events did not differ significantly between the two groups across the duration of the trial. The results of the primary endpoint analyses in the prespecified subgroups were consistent with those in the overall population (Fig. S2 in the Supplementary Appendix).

During the first 12 months of follow-up, 88 patients in the CTA group, as compared with 91 in the functional-testing group, had a primary endpoint event (hazard ratio, 0.94; 95\% CI, 0.70 to $1.26 ; \mathrm{P}=0.68$ ) (Table S4 in the Supplementary Appendix). At 12 months, the secondary end point of the composite of the primary end point plus invasive cardiac catheterization showing no obstructive CAD occurred in 256 patients (5.1\%) in the CTA group, as compared with 296 (5.9\%) in the functional-testing group (hazard ratio, $0.85 ; 95 \%$ CI, 0.72 to $1.00 ; \mathrm{P}=0.06)$. At 12 months, the risk of death or nonfatal myocardial infarction was lower in the CTA group than in the functional-testing group (hazard ratio, 0.66 ; $95 \%$ $\mathrm{CI}, 0.44$ to $1.00 ; \mathrm{P}=0.049$ ). Other prespecified end points were similar in the two study groups. There were 37 patients with mild safety events in the CTA group and 21 in the functional-testing group (Table S5 in the Supplementary Appendix). 


\section{CARDIAC CATHETERIZATION AND RADIATION DATA}

Overall, 1015 patients underwent at least one cardiac catheterization within 90 days after randomization: 609 of 4996 patients (12.2\%) in the CTA group and 406 of $5007(8.1 \%)$ in the functional-testing group. The results for 170 of the 609 patients (27.9\%) in the CTA group, as compared with 213 of the 406 (52.5\%) in the functional-testing group, showed no obstructive CAD. The secondary end point of catheterization showing no obstructive CAD occurred in $3.4 \%$ of the patients in the CTA group as compared with $4.3 \%$ of those in the functional-testing group $(\mathrm{P}=0.02)$. Revascularization was performed within 90 days after randomization in 311 of 4996 patients (6.2\%) in the CTA group as compared with 158 of $5007(3.2 \%)$ in the functional-testing group $(\mathrm{P}<0.001)$, including 72 patients and 38 patients, respectively, who underwent coronary-artery bypass grafting.

The distribution of cumulative radiation exposure at 90 days was complex, since $4.0 \%$ of the patients in the CTA group and $32.6 \%$ of those in the functional-testing group had no exposure to ionizing radiation (Fig. S3 in the Supplementary Appendix). The median exposure was lower in the CTA group than in the functional-testing group $(10.0 \mathrm{mSv}$ vs. $11.3 \mathrm{mSv}$ ), although the mean exposure was higher in the CTA group (12.0 mSv vs. 10.1 $\mathrm{mSv}$ ); the overall exposure was higher in the CTA group than in the functional-testing group $(\mathrm{P}<0.001)$. Among the 6781 patients whom physicians intended to refer for nuclear stress testing if the patients were randomly assigned to the functional-testing strategy, the cumulative radiation exposure was lower in the CTA group (median, $10.1 \mathrm{mSv}$ [interquartile range, 5.7 to 17.1]; mean, $12.0 \mathrm{mSv}$ ) than in the functional-testing group (median, $12.6 \mathrm{mSv}$ [interquartile range, 11.1 to 16.0 ]; mean, $14.1 \mathrm{mSv})(\mathrm{P}<0.001)$.

\section{DISCUSSION}

PROMISE enrolled a large, community-based population of symptomatic patients undergoing evaluation for suspected $\mathrm{CAD}$ for whom noninvasive testing was indicated according to current guidelines and for whom a preferred strategy for the selection of the noninvasive test has not been established. Despite the presence of an intermediate level of risk, as estimated by historically validated models, the event rate was lower than predicted. A strategy of anatomical testing with the use of CTA, as compared with use of functional testing, did not reduce the incidence of events over a median follow-up of 25 months.

Advances in cardiovascular imaging have enhanced physicians' diagnostic abilities, but an increase in the use of advanced imaging methods without a clear demonstration of an effect on health outcomes has led to increased regulation that has been designed to control spending and improve quality. ${ }^{25}$ Together, the limited available data, a multiplicity of testing options, concerns regarding current patterns, ${ }^{5-8}$ and the introduction of CTA for the evaluation of CAD have resulted in calls for an improved evidence base for imaging as well as a paradigm shift from a focus on test performance to a focus on clinical end points to better determine the role of noninvasive testing in the evaluation of CAD symptoms. ${ }^{14,25,26}$ These concerns were addressed by several features of this trial, including its large size, randomized comparison of testing strategies, broadly representative community setting, use of clinical events as the primary end point, and inclusion of other relevant clinical outcomes as secondary end points. 
Both CTA and functional testing in this trial resulted in a primary event rate of $3.1 \%$ overall. This result is congruent with the event rate observed in an administrative data set of younger persons $(<65 \text { years of age })^{27}$ but not in the Euro Heart Survey of stable angina, which involved 3000 patients with a risk-factor profile that was similar to that in our cohort and in which the rate of death or myocardial infarction at 1 year was $2.3 \%{ }^{28}$ The low rate of endpoint events observed in the present trial may be due to the higher use of cardiovascular medications, especially statins, in our patients, as well as to other improvements in cardiovascular care over the past decade. Regardless of the cause, the event rate observed in our study probably reflects an excellent prognosis for patients with similar, new-onset, stable chest pain in real-world settings in which contemporary testing methods are used. Showing a difference in patient outcomes with different testing strategies given this excellent midterm prognosis would require a large incremental test effect driving differences in downstream care or an extremely large study sample.

As compared with functional testing, the CTA strategy was associated with a lower incidence of invasive catheterization showing no obstructive CAD during the 90 days after randomization, which was a prespecified secondary end point. Although more patients randomly assigned to CTA underwent at least one cardiac catheterization within 90 days after randomization (12.2\%, vs. $8.1 \%$ in the functional-testing group) and more patients in the CTA group underwent revascularization overall (6.2\% vs. 3.2\%), including coronaryartery bypass grafting, revascularization was not a trial end point. The lack of discernible differences in outcomes at 2 years is consistent with a trial that was not designed or powered to assess the effect of additional diagnostic tests (e.g., invasive catheterization) or specific therapeutic procedures (e.g., revascularization) on outcomes.

The mix of tests in the functional-testing group, including a test with and two tests without radiation exposure, created a complex picture of lower median but higher mean radiation exposures in the CTA group than in the functional-testing group. Since the choice of noninvasive test will dramatically alter relative exposures, the precise mix used in this trial is most useful for providing a snapshot of current practice across our enrolling sites, rather than offering definitive insights regarding harm or benefit from a CTA strategy versus a functional-testing strategy. However, if the choice for an individual patient is between CTA and nuclear stress testing, as was the case in $67.8 \%$ of our patients, then the exposures within the stratum of patients for whom nuclear stress testing was intended if they would be assigned to functional testing become most relevant. For these patients, the median cumulative exposure was $2.5 \mathrm{mSv}$ lower and the mean exposure $2.1 \mathrm{mSv}$ lower in the CTA group than in the functional-testing group. As expected, radiation exposure was much higher in the CTA group than in the functional-testing group in the stratum of patients for whom stress ECG or stress echocardiography was intended if they would be assigned to functional testing.

The radiation results should be interpreted in the context of the pragmatic trial design of PROMISE, which included minimal requirements for all testing methods, as noted previously. Thus, there was variation across sites with respect to radiation exposures in the CTA and nuclear scans owing to expected differences in equipment, isotopes, and image- 
acquisition protocols; adherence to best practices can be expected to result in even lower exposures with either of the two tests.

Despite the fact that the trial did not require a substantial risk-factor burden for enrollment, our population had the desired intermediate pretest likelihood of obstructive CAD of $53.3 \%,{ }^{2}$ indicating that the trial enrolled a target population that was highly suitable for an evaluation of the effectiveness of noninvasive testing in suspected CAD. These data also provide evidence of appropriate (rather than excessive) referral to testing. In spite of this, there was a low rate of test positivity in the two study groups, which is congruent with recent reports. ${ }^{5,29,30}$ These findings highlight a substantial opportunity to improve the selection of patients for noninvasive testing beyond currently accepted approaches. ${ }^{31}$

The pragmatic design of this trial enhances its generalizability to real-world settings but may limit applicability in settings with tightly controlled population selection, expert noninvasive test performance, or rigorously followed algorithms for subsequent care. This article does not address test performance or utility. Since the intended choice of functional test was used to stratify randomization and adjust the primary results, our findings are valid for a different mix of functional-testing methods.

In conclusion, in symptomatic patients with suspected CAD who required noninvasive testing, an initial strategy of CTA was not associated with better clinical outcomes than functional testing over a median follow-up of 2 years.

\section{Supplementary Material}

Refer to Web version on PubMed Central for supplementary material.

\section{Acknowledgments}

The views expressed in this article are those of the authors, who are solely responsible for the design and conduct of this study, all the study analyses, the drafting and editing of earlier versions of the manuscript, and its final contents. These views do not necessarily represent the official views of the National Heart, Lung, and Blood Institute (NHLBI).

Supported by grants from the NHLBI (R01HL098237, R01HL098236, R01HL098305 and R01HL098235).

Dr. Douglas reports receiving grant support from HeartFlow; Dr. Hoffmann, receiving grant support from Siemens Healthcare and HeartFlow; Dr. Patel, receiving fees for serving on advisory boards from AstraZeneca, Bayer, and Otsuka Pharmaceutical and grant support from HeartFlow, Janssen Pharmaceuticals, Johnson \& Johnson, and AstraZeneca; Dr. Mark, receiving personal fees from Medtronic, CardioDx, and St. Jude Medical and grant support from Eli Lilly, Bristol-Myers Squibb, Gilead Sciences, AGA Medical, Merck, Oxygen Biotherapeutics, and AstraZeneca; Dr. Krucoff, receiving personal fees from Abbott Vascular, Angel Medical Systems, Biosensors International, Medtronic, OrbusNeich, Svelte Medical Systems, Terumo, and Volcano and grant support from Abbott Vascular, Angel Medical Systems, Cardiovascular Systems, Eli Lilly, Ikaria, Medtronic, OrbusNeich, and Terumo; and Dr. Velasquez, receiving consulting fees from Alnylam Pharmaceuticals and Novartis and grant support from Abbott.

We thank all the patients who participated in this trial, and Sarah Hayden, Peter Hoffmann, and Beth Martinez for contributions to the study. 


\section{References}

1. Ladapo JA, Blecker S, Douglas PS. Physician decision making and trends in the use of cardiac stress testing in the United States: an analysis of repeated cross-sectional data. Ann Intern Med. 2014; 161:482-90. [PubMed: 25285541]

2. Fihn SD, Gardin JM, Abrams J, et al. 2012 ACCF/AHA/ACP/AATS/PCNA/SCAI/STS Guideline for the diagnosis and management of patients with stable ischemic heart disease: a report of the American College of Cardiology Foundation/American Heart Association Task Force on Practice Guidelines, and the American College of Physicians, American Association for Thoracic Surgery, Preventive Cardiovascular Nurses Association, Society for Cardiovascular Angiography and Interventions, and Society of Thoracic Surgeons. J Am Coll Cardiol. 2012; 60(24):e44-e164. [PubMed: 23182125]

3. National Institute for Health and Care Excellence. Management of stable angina: NICE clinical guideline 126. Dec. 2012 http://www.nice.org.uk/guidance/cg126/resources/guidance-managementof-stable-angina-pdf

4. Montalescot G, Sechtem U, Achenbach S, et al. 2013 ESC guidelines on the management of stable coronary artery disease: the Task Force on the management of stable coronary artery disease of the European Society of Cardiology. Eur Heart J. 2013; 34:2949-3003. [PubMed: 23996286]

5. Rozanski A, Gransar H, Hayes SW, et al. Temporal trends in the frequency of inducible myocardial ischemia during cardiac stress testing: 1991 to 2009. J Am Coll Cardiol. 2013; 61:1054-65. [PubMed: 23473411]

6. Patel MR, Peterson ED, Dai D, et al. Low diagnostic yield of elective coronary angiography. N Engl J Med. 2010; 362:886-95. Erratum, N Engl J Med 2010;363:498. [PubMed: 20220183]

7. Patel MR, Dai D, Hernandez AF, et al. Prevalence and predictors of nonobstructive coronary artery disease identified with coronary angiography in contemporary clinical practice. Am Heart J. 2014; 167:846-52. [PubMed: 24890534]

8. Douglas PS, Patel MR, Bailey SR, et al. Hospital variability in the rate of finding obstructive coronary artery disease at elective, diagnostic coronary angiography. J Am Coll Cardiol. 2011; 58:801-9. [PubMed: 21835315]

9. Budoff MJ, Dowe D, Jollis JG, et al. Diagnostic performance of 64-multi-detector row coronary computed tomographic angiography for evaluation of coronary artery stenosis in individuals without known coronary artery disease: results from the prospective multicenter ACCURACY (Assessment by Coronary Computed Tomographic Angiography of Individuals Undergoing Invasive Coronary Angiography) trial. J Am Coll Cardiol. 2008; 52:1724-32. [PubMed: 19007693]

10. Meijboom WB, Meijs MF, Schuijf JD, et al. Diagnostic accuracy of 64-slice computed tomography coronary angiography: a prospective, multicenter, multivendor study. J Am Coll Cardiol. 2008; 52:2135-44. [PubMed: 19095130]

11. Miller JM, Rochitte CE, Dewey M, et al. Diagnostic performance of coronary angiography by 64row CT. N Engl J Med. 2008; 359:2324-36. [PubMed: 19038879]

12. Min JK, Dunning A, Lin FY, et al. Age- and sex-related differences in all-cause mortality risk based on coronary computed tomography angiography findings results from the International Multicenter CONFIRM (Coronary CT Angiography Evaluation for Clinical Outcomes: An International Multicenter Registry) of 23,854 patients without known coronary artery disease. J Am Coll Cardiol. 2011; 58:849-60. [PubMed: 21835321]

13. Mark DB, Berman DS, Budoff MJ, et al. ACCF/ACR/AHA/NASCI/SAIP/SCAI/SCCT 2010 expert consensus document on coronary computed tomographic angiography: a report of the American College of Cardiology Foundation Task Force on Expert Consensus Documents. J Am Coll Cardiol. 2010; 55:2663-99. [PubMed: 20513611]

14. Douglas PS, Taylor A, Bild D, et al. Outcomes research in cardiovascular imaging: report of a workshop sponsored by the National Heart, Lung, and Blood Institute. JACC Cardiovasc Imaging. 2009; 2:897-907. [PubMed: 19608141]

15. Shaw L, Narula J. From adequate evidence to optimal evidence. JACC Cardiovasc Imaging. 2012; 5:1292-3. [PubMed: 23236983] 
16. Douglas PS, Hoffmann U, Lee KL, et al. PROspective Multicenter Imaging Study for Evaluation of chest pain: rationale and design of the PROMISE trial. Am Heart J. 2014; 167:796-803. [PubMed: 24890527]

17. Hicks KA, Tcheng JE, Bozkurt B, et al. 2014 ACC/AHA key data elements and definitions for cardiovascular endpoint events in clinical trials: a report of the American College of Cardiology/ American Heart Association Task Force on Clinical Data Standards (Writing Committee to Develop Cardiovascular Endpoints Data Standards). J Am Coll Cardiol. 2014 Dec 29. Epub ahead of print.

18. Gerber TC, Carr JJ, Arai AE, et al. Ionizing radiation in cardiac imaging: a science advisory from the American Heart Association Committee on Cardiac Imaging of the Council on Clinical Cardiology and Committee on Cardiovascular Imaging and Intervention of the Council on Cardiovascular Radiology and Intervention. Circulation. 2009; 119:1056-65. [PubMed: 19188512]

19. Mettler FA Jr, Huda W, Yoshizumi TT, Mahesh M. Effective doses in radiology and diagnostic nuclear medicine: a catalog. Radiology. 2008; 248:254-63. [PubMed: 18566177]

20. Chen J, Einstein AJ, Fazel R, et al. Cumulative exposure to ionizing radiation from diagnostic and therapeutic cardiac imaging procedures: a population-based analysis. J Am Coll Cardiol. 2010; 56:702-11. [PubMed: 20619569]

21. Cox DR. Regression models and life-tables. J R Stat Soc B. 1972; 34:187-220.

22. Kaplan EL, Meier P. Nonparametric estimation from incomplete observations. J Am Stat Assoc. 1958; 53:457-81.

23. Grundy SM, Cleeman JI, Daniels SR, et al. Diagnosis and management of the metabolic syndrome: an American Heart Association/National Heart, Lung, and Blood Institute Scientific Statement. Circulation. 2005; 112:2735-52. Errata, Circulation 2005;112(7):e297, e298. [PubMed: 16157765]

24. Goff DC Jr, Lloyd-Jones DM, Bennett G, et al. 2013 ACC/AHA guideline on the assessment of cardiovascular risk: a report of the American College of Cardiology/American Heart Association Task Force on Practice Guidelines. Circulation. 2014; 129(Suppl 2):S49-S73. Erratum, Circulation 2014;129:Suppl 2:S74-S75. [PubMed: 24222018]

25. Iglehart JK. Health insurers and medical-imaging policy - a work in progress. N Engl J Med. 2009; 360:1030-7. [PubMed: 19264694]

26. Shaw LJ, Min JK, Hachamovitch R, et al. Cardiovascular imaging research at the crossroads. JACC Cardiovasc Imaging. 2010; 3:316-24. [PubMed: 20223430]

27. Mudrick DW, Cowper PA, Shah BR, et al. Downstream procedures and outcomes after stress testing for chest pain without known coronary artery disease in the United States. Am Heart J. 2012; 163:454-61. [PubMed: 22424017]

28. Daly CA, De Stavola B, Sendon JL, et al. Predicting prognosis in stable angina — results from the Euro Heart Survey of stable angina: prospective observational study. BMJ. 2006; 332:262-7. [PubMed: 16415069]

29. Thomas GS, Voros S, McPherson JA, et al. A blood-based gene expression test for obstructive coronary artery disease tested in symptomatic nondiabetic patients referred for myocardial perfusion imaging the COMPASS study. Circ Cardiovasc Genet. 2013; 6:154-62. [PubMed: 23418288]

30. Muhlestein JB, Lappé DL, Lima JA, et al. Effect of screening for coronary artery disease using CT angiography on mortality and cardiac events in high-risk patients with diabetes: the FACTOR-64 randomized clinical trial. JAMA. 2014; 312:2234-43. [PubMed: 25402757]

31. Cheng VY, Berman DS, Rozanski A, et al. Performance of the traditional age, sex, and angina typicality-based approach for estimating pretest probability of angiographically significant coronary artery disease in patients undergoing coronary computed tomographic angiography: results from the multinational Coronary CT Angiography Evaluation for Clinical Outcomes: An International Multicenter Registry (CONFIRM). Circulation. 2011; 124:2423-32. [PubMed: 22025600] 


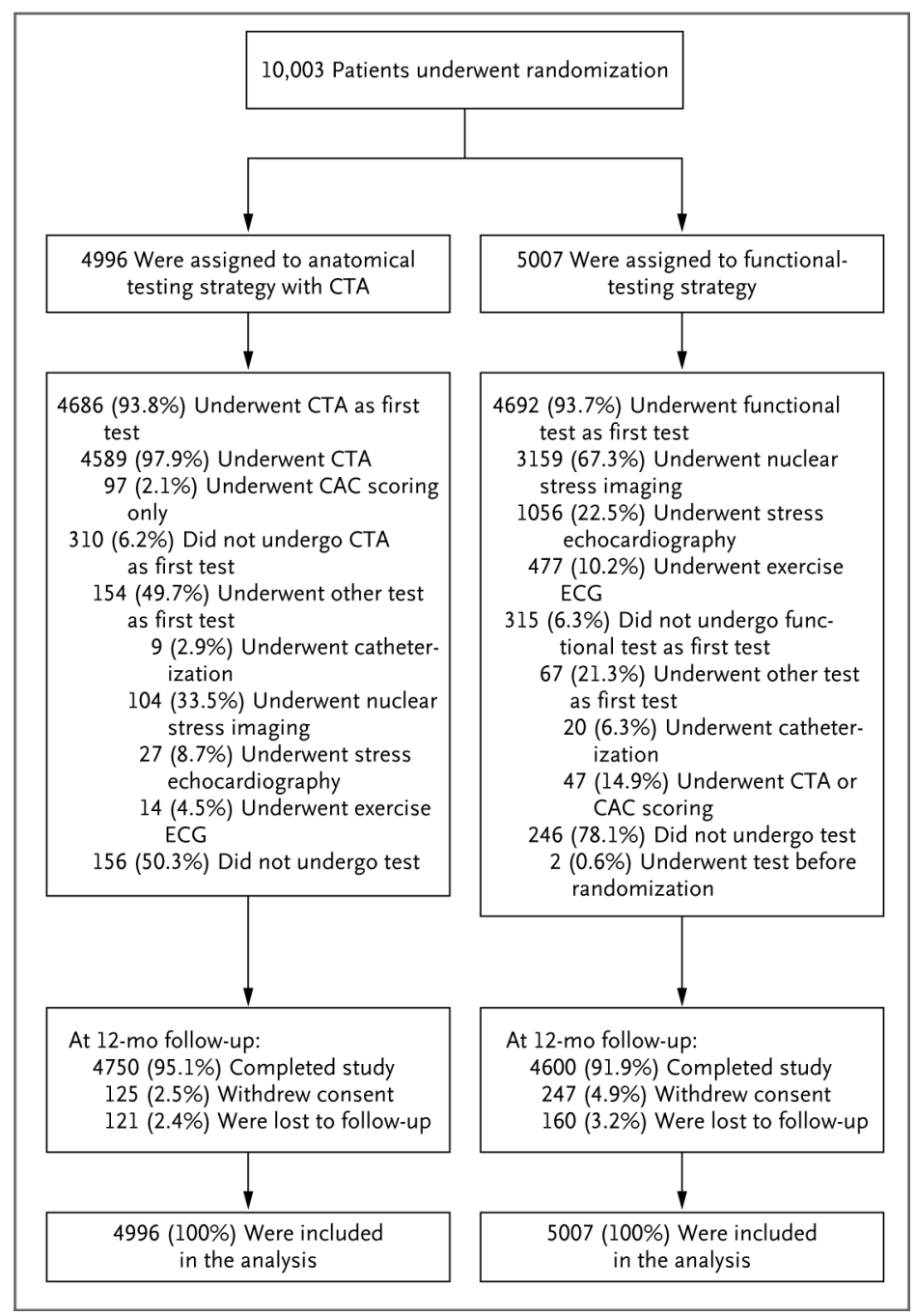

Figure 1. Enrollment, Randomization, and Follow-up of the Trial Patients

A total of 404 patients (248 patients in the functional-testing group and 156 in the computed tomographic angiography [CTA] group) did not undergo any testing owing to withdrawal from the study (26.5\% of the untested patients), missed appointment $(25.7 \%)$, financial hardship (21.8\%), medical reasons $(9.9 \%)$, scheduling conflicts $(7.9 \%)$, or miscellaneous reasons $(8.2 \%)$. Two patients underwent functional testing before randomization, and their tests were excluded. Data on all 10,003 patients were included in the analysis of the effectiveness of the testing strategy. Percentages may not sum to 100 owing to rounding. CAC denotes coronary-artery calcium, and ECG electrocardiography. 


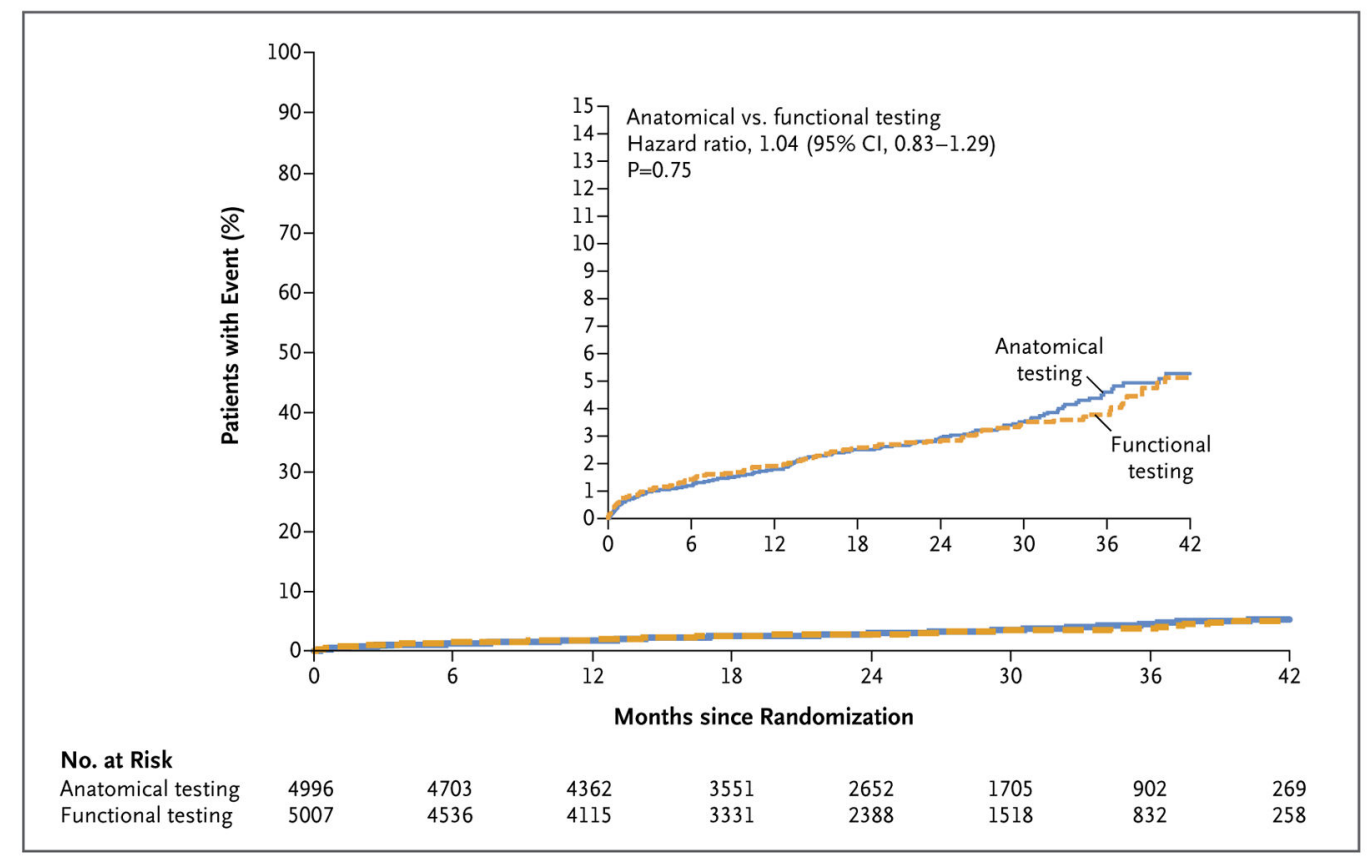

Figure 2. Kaplan-Meier Estimates of the Composite Primary End Point as a Function of Time after Randomization

The graph shows the unadjusted Kaplan-Meier estimates of the primary composite end point (death from any cause, nonfatal myocardial infarction, hospitalization for unstable angina, or major procedural complication). The adjusted hazard ratio for a CTA strategy, as compared with a usual-care strategy of functional testing, was 1.04 (95\% CI, 0.83 to 1.29), with adjustment for age, sex, risk equivalent of coronary artery disease (history of diabetes, peripheral arterial disease, or cerebrovascular disease), and the prespecification of the intended functional test if the patient were to be randomly assigned to the functional-testing group. The inset shows the same data on an enlarged y axis. 


\section{Table 1}

Characteristics of the Trial Participants at Baseline, According to Study Group.

\begin{tabular}{|c|c|c|}
\hline Characteristic & CTA Strategy $(N=4996)$ & $\begin{array}{c}\text { Functional-Testing Strategy }(\mathrm{N} \\
=\text { = 5007) }\end{array}$ \\
\hline Mean age $-\mathrm{yr}$ & $60.7 \pm 8.3$ & $60.9 \pm 8.3$ \\
\hline Female sex — no. $(\%)$ & $2595(51.9)$ & $2675(53.4)$ \\
\hline Racial or ethnic minority group - no./total no. $(\%)^{\dagger}$ & $1166 / 4968(23.5)$ & $1082 / 4973(21.8)$ \\
\hline \multicolumn{3}{|l|}{ Cardiac risk factor } \\
\hline Mean body-mass index & $30.5 \pm 6.1$ & $30.5 \pm 6.1$ \\
\hline Hypertension - no. (\%) & $3247(65.0)$ & $3254(65.0)$ \\
\hline Diabetes - no. $(\%)$ & $1065(21.3)$ & $1079(21.5)$ \\
\hline Dyslipidemia — no./total no. (\%) & $3365 / 4995(67.4)$ & $3402 / 5007(67.9)$ \\
\hline Family history of premature CAD - no./total no. $(\%)^{\S}$ & $1624 / 4979(32.6)$ & $1578 / 4991(31.6)$ \\
\hline Peripheral arterial or cerebrovascular disease - no. (\%) & $263(5.3)$ & $289(5.8)$ \\
\hline CAD risk equivalent - no. $(\%)^{q /}$ & $1246(24.9)$ & $1285(25.7)$ \\
\hline Metabolic syndrome - no. $(\%) \|$ & $1867(37.4)$ & $1905(38.0)$ \\
\hline Current or past tobacco use - no./total no. (\%) & 2533/4994 (50.7) & $2571 / 5006(51.4)$ \\
\hline Sedentary lifestyle - no./total no. $(\%)^{* *}$ & $2429 / 4985(48.7)$ & $2437 / 4997(48.8)$ \\
\hline History of depression - no. (\%) & $978(19.6)$ & $1080(21.6)$ \\
\hline \multicolumn{3}{|l|}{ Risk burden ${ }^{\dagger \dagger}$} \\
\hline No risk factors - no. $(\%)$ & $126(2.5)$ & $137(2.7)$ \\
\hline Mean no. of risk factors per patient & $2.4 \pm 1.1$ & $2.4 \pm 1.1$ \\
\hline $\begin{array}{l}\text { Mean combined Diamond and Forrester and Coronary Artery Surgery } \\
\text { Study risk score }\end{array}$ & $53.4 \pm 21.4$ & $53.2 \pm 21.4$ \\
\hline \multicolumn{3}{|l|}{ Relevant medication - no./total no. $(\%)$} \\
\hline Beta-blocker & $1205 / 4783(25.2)$ & $1194 / 4786(24.9)$ \\
\hline ACE inhibitor or ARB & $2089 / 4783(43.7)$ & $2105 / 4786(44.0)$ \\
\hline Statin & $2215 / 4783(46.3)$ & $2174 / 4786(45.4)$ \\
\hline Aspirin & $2164 / 4783(45.2)$ & $2116 / 4786(44.2)$ \\
\hline \multicolumn{3}{|l|}{ Primary presenting symptom - no./total no. $(\%)$} \\
\hline Chest pain & $3673 / 4992(73.6)$ & $3599 / 5004(71.9)$ \\
\hline Dyspnea on exertion & $712 / 4992(14.3)$ & $778 / 5004(15.5)$ \\
\hline Other $\S \S$ & $607 / 4992(12.2)$ & $627 / 5004(12.5)$ \\
\hline \multicolumn{3}{|l|}{ Type of angina - no. $(\%)^{I I I I}$} \\
\hline Typical & $590(11.8)$ & $576(11.5)$ \\
\hline Atypical & $3873(77.5)$ & $3900(77.9)$ \\
\hline Nonanginal pain & $533(10.7)$ & $531(10.6)$ \\
\hline
\end{tabular}


* Plus-minus values are means \pm SD. There were no significant between-group differences at baseline, except with respect to racial or ethnic minority group and history of depression. ACE denotes angiotensin-converting enzyme, ARB angiotensin-receptor blocker, CAD coronary artery disease, and CTA computed tomographic angiography.

${ }^{\dagger}$ Racial or ethnic minority group was self-reported, with the status of "minority" being defined by the patient.

${ }^{*}$ Body-mass index is the weight in kilograms divided by the square of the height in meters.

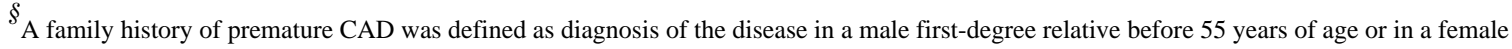
first-degree relative before 65 years of age.

${ }^{I}$ CAD risk equivalent was defined as diabetes, peripheral vascular disease, or cerebrovascular disease.

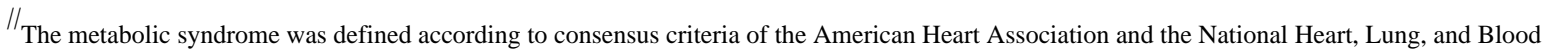
Institute. 23

$* *$

Sedentary lifestyle was defined by the patient as not participating in regular physical activities at least one time per week over the previous month.

${ }^{\dagger \dagger}$ Risk factors included hypertension, diabetes, dyslipidemia, family history of premature CAD, and tobacco use.

${ }^{+}$Combined Diamond and Forrester and Coronary Artery Surgery Study risk scores ${ }^{2}$ range from 0 to 100 , with higher scores indicating a greater likelihood of obstructive CAD.

$\S \S$ Other primary symptoms were (in descending order of frequency) fatigue or weakness, arm or shoulder pain, palpitations, dizziness or lightheadedness, and neck or jaw pain.

${ }^{I I I}$ The type of angina was reported by the study-site investigators. 


\section{Table 2}

End Points According to Study Group.*

\begin{tabular}{|c|c|c|c|c|}
\hline End Point & $\begin{array}{l}\text { CTA Strategy }(\mathrm{N} \\
=4996)\end{array}$ & $\begin{array}{l}\text { Functional-Testing } \\
\text { Strategy (N = 5007) }\end{array}$ & $\begin{array}{c}\text { Adjusted Hazard } \\
\text { Ratio }(95 \% \text { CI })\end{array}$ & P Value \\
\hline \multicolumn{5}{|l|}{ Clinical end point - no. of patients } \\
\hline Primary composite end point & 164 & 151 & $1.04(0.83-1.29)$ & 0.75 \\
\hline Death from any cause & 74 & 75 & & \\
\hline Nonfatal myocardial infarction & 30 & 40 & & \\
\hline Hospitalization for unstable angina & 61 & 41 & & \\
\hline Major procedural complication & 4 & 5 & & \\
\hline $\begin{array}{l}\text { Primary end point plus catheterization showing no } \\
\text { obstructive CAD }\end{array}$ & 332 & 353 & $0.91(0.78-1.06)$ & 0.22 \\
\hline Death or nonfatal myocardial infarction & 104 & 112 & $0.88(0.67-1.15)$ & 0.35 \\
\hline $\begin{array}{l}\text { Death, nonfatal myocardial infarction, or hospitalization } \\
\text { for unstable angina }\end{array}$ & 162 & 148 & $1.04(0.84-1.31)$ & 0.70 \\
\hline \multicolumn{5}{|l|}{ Test-related end point } \\
\hline $\begin{array}{l}\text { Invasive catheterization showing no obstructive CAD } \\
- \text { no. }(\%)\end{array}$ & $170(3.4)$ & $213(4.3)$ & - & 0.02 \\
\hline \multicolumn{5}{|l|}{$\begin{array}{l}\text { Cumulative radiation exposure in all procedures } ₫ 0 \\
\text { days after randomization }-\mathrm{mSv}\end{array}$} \\
\hline All patients & $12.0 \pm 8.5$ & $10.1 \pm 9.0$ & - & $<0.001$ \\
\hline Median & 10.0 & 11.3 & & \\
\hline Interquartile range & $5.6-17.2$ & $0.0-13.5$ & & \\
\hline \multicolumn{5}{|l|}{ Intended functional test before randomization } \\
\hline Nuclear stress testing & $12.0 \pm 8.4$ & $14.1 \pm 7.6$ & - & $<0.001$ \\
\hline Median & 10.1 & 12.6 & & \\
\hline Interquartile range & $5.7-17.1$ & $11.1-16.0$ & & \\
\hline Stress echocardiography & $12.6 \pm 9.0$ & $1.3 \pm 4.3$ & - & $<0.001$ \\
\hline Median & 10.6 & 0.0 & & \\
\hline Interquartile range & $5.5-18.3$ & $0.0-0.0$ & & \\
\hline Exercise electrocardiography & $10.4 \pm 7.8$ & $2.3 \pm 5.4$ & - & $<0.001$ \\
\hline Median & 8.5 & 0.0 & & \\
\hline Interquartile range & $4.8-15.7$ & $0.0-0.0$ & & \\
\hline
\end{tabular}

* Plus-minus values are means $\pm \mathrm{SD}$. A patient may have had more than one of the component events that make up the composite end point, in which case the patient is included in the count for each individual component event that occurred. Hazard ratios were adjusted for age, sex, CAD risk equivalent (history of diabetes, peripheral arterial disease, or cerebrovascular disease), and the prespecification of the intended functional test if the patient were to be randomly assigned to the functional-testing group. Test-related end points were not adjusted. 